\title{
SIMULACIÓN DE UN ESCURRIMIENTO REACTIVO AL INTERIOR DE UNA CÁMARA DE COMBUSTIÓN
}

\section{REACTIVE FLUID FLOW SIMULATION INSIDE COMBUSTION CHAMBER}

\author{
Carlos H. Salinas Lira ${ }^{1} \quad$ Pedro A. Pacheco Fernandoy ${ }^{1}$ \\ Recibido 28 de abril de 2006, aceptado 16 de enero de 2007 \\ Received: April 28, 2006 Accepted: January 16, 2007
}

\begin{abstract}
RESUMEN
El presente trabajo dice relación con la simulación de gases calientes al interior de una cámara de tubular combustión. Siendo así, se generan mallas en dominios tubulares curvilíneos. La inyección de combustible es realizada a través de un cuerpo esférico posicionado en el eje de simetría de la cámara afectado por un flujo primario axial y un flujo secundario radial. El fluido es considerado newtoniano incompresible y con propiedades termofísicas constantes, en cuanto el flujo es considerado laminar, modelado a través de la ecuaciones de Navier-Stokes. La formulación de Shvab-Zel'dovich es utilizada para modelar el transporte de energía y especies a través de una variable denominada Potencial de Acoplamiento. El modelo matemático es resuelto numéricamente a través del Método de los Volúmenes Finitos descrito en coordenadas curvilíneas con arreglo colocalizado de variables. Los términos difusivos son representados por diferencias centradas y se usa el esquema WUDS para los términos convectivos. La integración temporal es del tipo implícito. Resultados de campos de velocidades, temperaturas y concentraciones son mostrados y comparados con datos encontrados en la literatura especializada. Se concluye en cuanto a la calidad cualitativa y cuantitativa de los resultados generados y en particular en lo que dice relación con la forma de la llama.
\end{abstract}

Palabras clave: Simulación, combustión, volúmenes finitos, cámara, modelo numérico.

\section{ABSTRACT}

The present work is related to the simulation of hot gases inside a tubular combustion chamber. This way, meshes are generated in tubular curvilinear domains. The fuel injection is carried out through a spherical body positioned in the chamber symmetry axis, affected by an axial primary flow and a radial secondary flow. The fluid is considered Newtonian incompressible, with constant thermo physics properties. In regard to the fluid flow, this is considered laminar, modeled through Navier-Stokes Equations. The Shvab-Zel'dovich formulation is used to model energy and species transport through a variable denominated Linkage Potential. The mathematical model is solved numerically through the Finite Volumes Method in a curvilinear coordinated system, with co-located variable arrangement. Centered differences and $W U D S$ schemes are used to represent the diffusion in convective terms. The temporary integration is of the implicit type. Results of fluid flow fields, temperatures and concentrations are shown and compared with specialized literature data. Conclusions are drawn from quality and quantitative results according to the available data, particularly, regarding flame shape.

Keywords: Simulation, combustion, finite volumes, chamber, numerical model.

\section{INTRODUCCIÓN}

El estudio de la combustión ha sido unos de los fenómenos de la naturaleza más complejos de abordar, debido al fuerte acoplamiento de los fenómenos físicos y químicos involucrados, como también a las condiciones de operación en las cuales se encuentra frecuentemente expuesta. Soluciones analíticas son escasas y presentan múltiples restricciones. Estas soluciones están principalmente dedicadas para fines académicos y, por lo tanto, son de poca aplicación práctica en ingeniería [1].

\footnotetext{
1 Departamento de Ingeniería Mecánica. Universidad del Bío-Bío. Av. Collao 12002. Concepción, Chile. Fono: (56-41) 731455/731499, fax: (56-41) 731022.E-mail: casali@ubiobio.cl,ppachecofernandoy@yahoo.es
} 
Desde la aparición de los computadores digitales en conjunto con la ciencia de Dinámica de los Fluidos Computacional $\mathrm{CFD}^{2}$, ha sido posible obtener una noción de los fenómenos físicos que ocurren en varias aplicaciones de ingeniería a través de la solución de las ecuaciones de transporte que rigen dichos fenómenos. Sin embargo, su aplicación a problemas de interés prácticos de la combustión ha sido menos exitoso, debido a la complejidad del proceso, esto es: la tasa de reacción química en cadena, descrita por la ley de Arrhenius, resulta en una cantidad de ecuaciones prohibitivas para la modelación. Además existe un fuerte acoplamiento entre las ecuaciones de acuerdo con el modelo de Arrhenius que describe la velocidad de reacción en función de la temperatura $[2,3]$, acoplando de esta forma la ecuación de energía con las ecuaciones de especies. De acuerdo a lo anterior, los problemas de combustión son abordados generalmente a través de la solución de una única ecuación de transporte, como el modelo de Shvab-Zel'dovich [1,2] para llamas no premezcladas. Las hipótesis de esta formulación son: Primero, la reacción ocurre infinitamente rápida y, por lo tanto, se tiene un único paso de reacción. Segundo, se considera un número de Lewis unitario para combustible y oxidante. Tercero, por último, se aplica la hipótesis de llama fina, presentándose ésta como una discontinuidad de derivada para los campos de temperatura, y fracción másica de combustible y oxidante. Según Katta et al [2], la formulación de Shvab-Zel'dovich entrega con precisión la posición y forma de la llama, así como la distribución de temperaturas en el lado del oxidante, debido a que el número de Lewis en el lado del oxidante es aproximadamente unitario. Sin embargo, este método no describe los fenómenos de ignición, extinción, y formación de contaminantes. Para analizar estos fenómenos, se debe realizar el estudio detallado de las reacciones en cadena usando la ley de Arrhenius. Este abordaje se conoce como Química Finita.

Por otro lado, el proceso de mezcla de especies reactivas es en régimen turbulento y, además, el flujo está confinado generalmente a geometrías complejas. De esto último surge la necesidad de resolver las ecuaciones en mallas bien finas, pues las aproximaciones clásicas para resolver la turbulencia, como el modelo k-e, resultan poco aplicables debido a la deficiencia que presentan estos métodos para capturar las pronunciadas curvaturas de las líneas de corriente presentes en estos flujos [3-5]. El método más exacto es el $\mathrm{DNS}^{3}$, pero resulta prohibitivo de aplicar a situaciones de interés práctico $[3,4]$. El método que ha surgido con mayor énfasis en los últimos años es el LES

2 Del acrónimo Computational Fluid Dynamics

3 Del acrónimo Direct Numerical Simulation
[3-5] incluidos problemas de combustión. El tratamiento de la combustión usado en estos trabajos consiste en un conjunto de formulaciones tipo Shvab-Zel'dovich como el modelo Flamelet Laminar para llamas premezcladas y no premezcladas [3, 4], y el modelo de la Ecuación $\mathrm{G}$ para llamas premezcladas [5].

En el presente trabajo se modela combustión con régimen de flujo laminar, y con propiedades termo físicas constantes. La formulación de Shvab-Zel'dovich es usada para resolver el fenómeno reactivo. La posición y forma de la llama es de particular interés, pues de esta forma se estima si la combustión fue completa o no, y si la llama afecta directamente las paredes de la cámara de combustión. Por lo tanto, la solución de la variable denominada Potencial de Acoplamiento será utilizada para determinar la posición y forma de la llama.

\section{GENERACIÓN DE MALLAS}

La técnica de generación numérica de mallas basada en la solución de un sistema de ecuaciones de Poisson, ver Thompson [6], fue adoptada, con esquema iterativo de Hilgenstock [7], para el control de la densidad y forma de las coordenadas curvilíneas, a través de las funciones de control de malla, $P(\xi, \eta)$ y $Q(\xi, \eta)$. El método de Hilgenstock establece como condiciones a satisfacer, el espaciamiento y el ángulo de intersección de las coordenadas adyacentes al contorno. Normalmente un sistema de coordenadas ortogonal deseado.

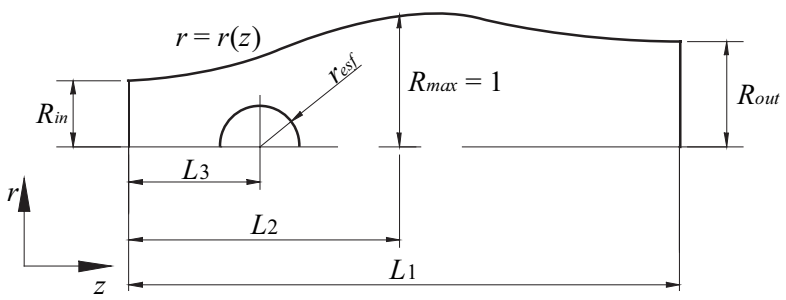

Figura 1. Geometría de la Cámara de Combustión a Modelar.

La malla es generada en el dominio cartesiano $(x, y)$ a partir de un sistema de coordenadas arbitrado en el dominio computacional $(\xi, \eta)[6,7]$, esto es:

$$
\begin{array}{r}
\alpha \frac{\partial^{2} x}{\partial \xi^{2}}-2 \beta \frac{\partial^{2} x}{\partial \eta \partial \xi}+\gamma \\
\frac{\partial^{2} x}{\partial \eta^{2}}+J^{2} P(\xi, \eta) \frac{\partial x}{\partial \xi} \\
+J^{2} Q(\xi, \eta) \frac{\partial x}{\partial \eta}=0
\end{array}
$$




$$
\begin{array}{r}
\alpha \frac{\partial^{2} y}{\partial \xi^{2}}-2 \beta \frac{\partial^{2} y}{\partial \eta \partial \xi}+\gamma \frac{\partial^{2} y}{\partial \eta^{2}}+J^{2} P(\xi, \eta) \frac{\partial y}{\partial \xi} \\
+J^{2} Q(\xi, \eta) \frac{\partial y}{\partial \eta}=0
\end{array}
$$

donde $\alpha, \beta$ y $\gamma$ son los coeficientes geométricos, dados por $\alpha=x_{\eta}{ }^{2}+y_{\eta}{ }^{2}, \beta=x_{\eta} x_{\xi}+y_{\eta} y_{\xi} \mathrm{y} \gamma=x_{\xi}{ }^{2}+y_{\xi}{ }^{2}$. Entretanto,

$J$ es el Jacobiano de la transformación de coordenadas, expresado por $J=x_{\xi} y_{\eta}-x_{\eta} y_{\xi}$, donde $x_{\eta}, x_{\xi}, y_{\eta}$ e $y_{\xi}$

representan las métricas de la transformación inversa [6]. Las funciones de control de malla, $P(\xi, \eta)$ y $Q(\xi, \eta)$, de acuerdo al esquema iterativo de Hilgenstock, son evaluadas como:

$$
P^{n+1}=P^{n}+\Delta P^{n} \quad Q^{n+1}=Q^{n}+\Delta Q^{n}
$$

donde $\Delta P^{n}$ y $\Delta Q^{n}$ son las correcciones necesarias para obtener $P(\xi, \eta)$ y $Q(\xi, \eta)$ cuyos valores satisfacen las condiciones de espaciamiento y ortogonalidad impuestas en los contornos de la malla. El símbolo $n$ indica el nivel de iteración. Los valores de $\Delta P^{n}$ y $\Delta Q^{n}$ son determinados de acuerdo con el tipo de malla requerida, pues los valores de $P(\xi, \eta)$ tienen influencia en las líneas coordenadas $\xi$, y los valores de $Q(\xi$, $\eta)$ en las líneas coordenadas $\eta$, como observado en la figura 2. La transformación de coordenadas requerida es mostrada en la figura 3.

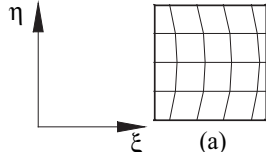

(a)

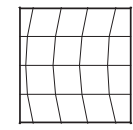

(b)

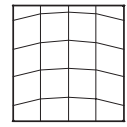

(c)

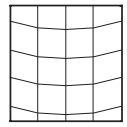

(d)
Figura 2. Efectos de $P(\xi, \eta)$ y $Q(\xi, \eta)$ en las líneas coordenadas de la malla. (a) y (b) $Q(\xi, \eta)=0$ y $P(\xi, \eta)>0$ y $P(\xi, \eta)<0$, (c) y (d) $P(\xi, \eta)=0$ y $Q(\xi, \eta)>0$ y $Q(\xi, \eta)<0$.

De acuerdo con la malla requerida, haciendo referencias a las figuras 2 y 3 , el control del espaciamiento para el contorno sur del dominio computacional (figura 3) será a través de los valores de $Q(\xi, \eta)$, en cuanto que el control del ángulo de intersección de las líneas coordenadas que llegan al contorno norte y sur del dominio computacional será a través de $P(\xi, \eta)$. Por lo tanto, las correcciones para obtener el espaciamiento y el ángulo de intersección deseado de la ecuación (3), son expresadas en la ecuación (4).
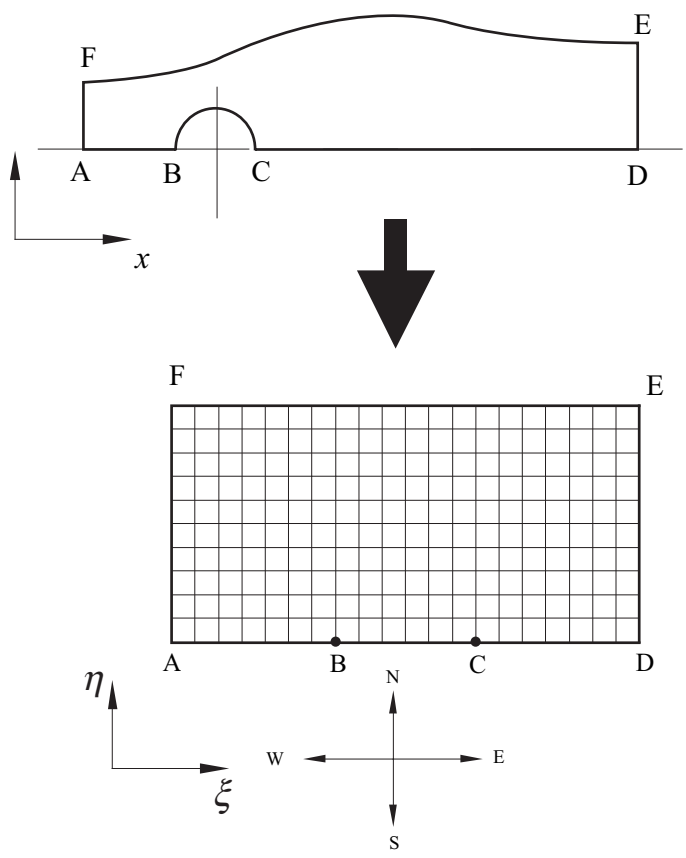

Figura 3. Transformación de coordenadas desde el dominio físico al computacional.

$\Delta Q^{n}=+\tan ^{-1}\left(\frac{\Delta s^{n}-\Delta s^{*}}{\Delta s^{*}}\right) \Delta P^{n}=+\tan ^{-1}\left(\frac{\alpha^{n}-\alpha^{*}}{\alpha^{*}}\right)$

donde $\Delta s^{*}$ y $\alpha^{*}$, son el espaciamiento, y el ángulo de intersección deseado, respectivamente. Entretanto $\Delta s^{n}$ y $\alpha^{n}$, son los valores reales de estas variables, las cuales son calculadas a través de la fórmula de distancia entre puntos $\left(\Delta s^{n}\right)$, y del producto escalar de vectores a través de la función arco coseno $\left(\alpha^{n}\right)$ [7]. Luego de obtener los valores de $P(\xi, \eta)$ y $Q(\xi, \eta)$ en los contornos, los valores en el dominio deben ser calculados. Hilgenstock [7] propone funciones de extrapolación para obtener los valores de $P(\xi, \eta)$ y $Q(\xi, \eta)$ para los puntos internos del dominio expresadas por:

$$
\begin{aligned}
& P(\xi, \eta)=P(\xi, 1) \exp \left[-a(\eta-1) /\left(\eta_{\max }-1\right)\right] \\
& +P\left(\xi, \eta_{\max }\right) \exp \left[-b(\eta-1) /\left(\eta_{\text {max }}-1\right)\right] \\
& Q(\xi, \eta)=Q(\xi, 1) \exp \left[-c(\eta-1) /\left(\eta_{\max }-1\right)\right] \\
& +Q\left(\xi, \eta_{\max }\right) \exp \left[-d(\eta-1) /\left(\eta_{\max }-1\right)\right]
\end{aligned}
$$

donde $a, b, c$ y $d$ son los coeficientes de decaimiento para los valores de $P(\xi, \eta)$ y $Q(\xi, \eta)$, en los contornos, que tienen por objetivo controlar la distribución de los puntos en el interior del dominio. 


\section{MODELO FÍSICO}

La figura 4 identifica las entradas y salidas de masa de la cámara de combustión a partir de las cuales se definen las condiciones de contorno del presente problema. La solución del campo de velocidades es realizada a través de las ecuaciones de Navier-Stokes en coordenadas cilíndricas, las cuales son presentadas en la forma conservativa y adimensional.

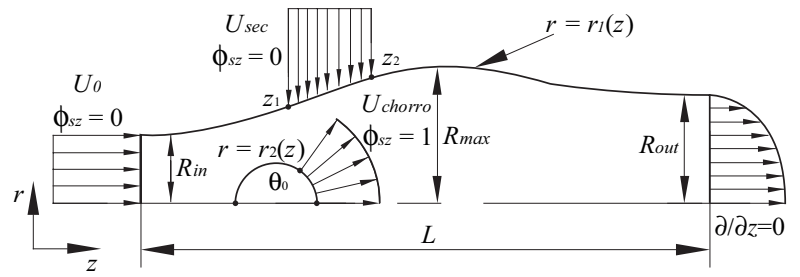

Figura 4. Condiciones de Contorno.

$$
\begin{gathered}
\frac{1}{r} \frac{\partial}{\partial r}\left(r u_{r}\right)+\frac{\partial u_{z}}{\partial z}=0 \\
\frac{\partial u_{z}}{\partial \tau}+\frac{\partial\left(u_{r} u_{z}\right)}{\partial r}+\frac{\partial\left(u_{z} u_{z}\right)}{\partial z}=-\frac{\partial P}{\partial z}+ \\
+\frac{1}{R e}\left[\frac{1}{r} \frac{\partial}{\partial r}\left(r \frac{\partial u_{z}}{\partial r}\right)+\frac{\partial}{\partial z}\left(\frac{\partial u_{z}}{\partial z}\right)\right] \\
\frac{\partial u_{r}}{\partial t}+\frac{\partial\left(u_{z} u_{r}\right)}{\partial z}+\frac{\partial\left(u_{r} u_{r}\right)}{\partial r}=-\frac{\partial p}{\partial r}+ \\
\frac{1}{R e}\left[\frac{1}{r} \frac{\partial}{\partial z}\left(r \frac{\partial u_{r}}{\partial z}\right)+\frac{1}{r} \frac{\partial}{\partial r}\left(r \frac{\partial u_{r}}{\partial r}\right)-\frac{u_{r}}{r^{2}}\right]
\end{gathered}
$$

donde $u_{r}$ y $u_{z}$ son las velocidades axial y radial, y $P$ es la presión. El número de Reynolds es definido como:

$$
R e=\frac{\rho U_{0} R_{\max }}{\mu}
$$

Entretanto, el fenómeno reactivo es modelado a través de la formulación de Shvab-Zel'dovich. Las ecuaciones que definen el proceso de combustión son la ecuación de conservación de energía, y la ecuación de conservación de especies para combustible y oxidante, las cuales son mostradas a continuación en la forma adimensional.

$$
\begin{aligned}
& \frac{\partial \theta}{\partial \tau}+\frac{\partial\left(u_{r} \theta\right)}{\partial r}+\frac{\partial\left(u_{z} \theta\right)}{\partial z} \\
& =\frac{1}{P e}\left[\frac{1}{r} \frac{\partial}{\partial r}\left(r \frac{\partial \theta}{\partial r}\right)+\frac{\partial}{\partial z}\left(\frac{\partial \theta}{\partial z}\right)\right]+\frac{\dot{\omega}_{f} \Delta H_{r}}{c_{p} \Delta T} \frac{R_{\max }}{\rho \cdot U} \\
& \frac{\partial Y_{f}}{\partial \tau}+\frac{\partial\left(u_{r} Y_{f}\right)}{\partial r}+\frac{\partial\left(u_{z} Y_{f}\right)}{\partial z} \\
& =\frac{1}{L e P e}\left[\frac{1}{r} \frac{\partial}{\partial r}\left(r \frac{\partial Y_{f}}{\partial r}\right)+\frac{\partial}{\partial z}\left(\frac{\partial Y_{f}}{\partial z}\right)\right]+\dot{\omega}_{f} \frac{R_{\max }}{\rho \cdot U} \\
& \quad \frac{\partial Y_{o}}{\partial \tau}+\frac{\partial\left(u_{r} Y_{o}\right)}{\partial r}+\frac{\partial\left(u_{z} Y_{o}\right)}{\partial z} \\
& =\frac{1}{L e P e}\left[\frac{1}{r} \frac{\partial}{\partial r}\left(r \frac{\partial Y_{o}}{\partial r}\right)+\frac{\partial}{\partial z}\left(\frac{\partial Y_{o}}{\partial z}\right)\right]+\dot{\omega}_{o} \frac{R_{\max }}{\rho \cdot U}
\end{aligned}
$$

donde $\theta$ es la temperatura adimensional dada por $\theta=\left(T-T_{f}\right) /\left(T_{o}-T_{f}\right)$ con $T_{o}$ siendo la temperatura del oxidante, y $T_{f}$ la temperatura del combustible. Entretanto, $Y_{f}$ e $Y_{o}$ son la fracción másica de combustible y oxidante. El número de Lewis y Peclet respectivamente son expresados por:

$$
\begin{gathered}
L e=\frac{k}{\rho C_{p} D_{i j}}=\frac{\alpha}{D_{i j}} \\
P e=\operatorname{Re} \operatorname{Pr}=\frac{U_{0} R_{\max }}{\alpha} \quad \operatorname{Pr}=\frac{v}{\alpha}
\end{gathered}
$$

Las ecuaciones (11), (12) y (13) son ecuaciones de convección difusión transiente y, por lo tanto, pertenecen a la misma familia de ecuaciones (Ecuaciones Parabólicas). La diferencia reside en el número de Lewis y el término fuente asociado a la reacción química. El primer paso consiste en hacer el número de Lewis unitario para combustible y oxidante. El segundo paso es eliminar el término fuente químico de las tres ecuaciones. Esto se hace por medio de la siguiente definición, válida para llamas no premezcladas:

$$
\begin{array}{r}
1 \mathrm{Kg} \text { Combustible }+s \text { Kg Oxidante } \rightarrow \\
(1+s) \text { Kg Productos }
\end{array}
$$

De esta relación se obtienen que las tasas de consumo de combustible y oxidante están dadas por: 


$$
\dot{\omega}_{f}=\frac{\dot{\omega}_{o}}{s}
$$

Por lo tanto, dividiendo ecuación (13) por $s$, y luego substrayendo este resultado a la ecuación (12), se obtiene el primer escalar conservado, expresado por

$$
\phi_{f o}=Y_{f}-\frac{Y_{o}}{s}
$$

Esta variable escalar es la más importante de las tres, pues cuando $\phi_{f_{0}}=0$ se identifica la posición de la superficie estequiométrica, la cual indica la zona de reacción, esto es, la posición de la llama $[1,8]$. Entretanto, el resto de los escalares son obtenidos de forma análoga, mostrados a continuación:

$$
\begin{gathered}
\phi_{t o}=\frac{J a}{s} Y_{o}-\theta \\
\phi_{t f}=J a \cdot Y_{f}-\theta
\end{gathered}
$$

donde $J a$ es el número de Jacob, expresado por:

$$
J a=\frac{\Delta H_{R}}{c_{p} \Delta T}
$$

Este número adimensional representa la relación entre la energía latente (entalpía de reacción) y la energía sensible (entalpía) que existe por la diferencia de temperatura entre el combustible y el oxidante en sus respectivas corrientes de entrada. Las Ecs. (19) y (20) son obtenidas como: Ec. (13)* $(\mathrm{Ja} / \mathrm{s})-$ Ec. (11) y Ec. (12)*Ja - Ec. (11), respectivamente.

Los escalares $\phi_{f o}, \phi_{t o} y \phi_{t f}$ son denominados escalares conservados. Para conocer las relaciones entre estos tres escalares conservados se hace uso del procedimiento de normalización, donde los valores de los 3 escalares varían de 0 a 1 . Cuando las condiciones de contorno de dos escalares conservados normalizados, en las corrientes de combustible y oxidante respectivamente, coincidan, la solución de estos dos escalares es la misma y, por lo tanto, la solución del tercer escalar conservado es obtenida de una ecuación que define la condición de acoplamiento [8]. La ecuación de normalización de variables es:

$$
\phi_{i}^{*}=\frac{\phi_{i}-\phi_{i, \min }}{\phi_{i, \max }-\phi_{i, \min }}
$$

Los valores máximos y mínimos se obtienen de acuerdo a las condiciones de contorno para cada ecuación a partir de la ecuaciones originales [8]. Siendo así, los tres escalares conservados y normalizados son:

$$
\begin{gathered}
\phi_{f o}^{*}=-\frac{s \cdot \phi_{t f}+Y_{O 2}}{s+Y_{O 2}} \quad \phi_{t f}^{*}=-\frac{\phi_{t f}-J a}{J a+1} \\
\phi_{t o}^{*}=\frac{s\left(\phi_{t o}+1\right)-Y_{O 2} J a}{s-Y_{O 2} J a}
\end{gathered}
$$

La posición de la línea estequiométrica para el primer escalar conservado normalizado es:

$$
\phi_{f o, s t}^{*}=\frac{Y_{O 2}}{s+Y_{O 2}}
$$

La condición de acoplamiento se obtiene de sumar las ecuaciones (19) y (20):

$$
\phi_{t o}+J a \cdot \phi_{f o}-\phi_{t f}=0
$$

De acuerdo con las condiciones de contorno normalizadas, se tiene que dos variables escalares tienen soluciones semejantes, al tener los mismos valores de condiciones de contorno en las corrientes de combustible y oxidante. Los escalares conservados normalizados idénticos son:

$$
\phi_{f o}^{*}=\phi_{t o}^{*}
$$

por lo tanto, $\phi_{f o}^{*}$ es seleccionada como la variable que será resuelta, y es definida como Potencial de Acoplamiento, con condiciones de contorno para la corriente de oxidante de $\phi_{f o}^{*}=0$, y para la corriente de combustible $\phi_{f o}^{*}=1$.

Para contornos sólidos la condición de contorno de impermeabilidad es aplicada, y para $r=0$, la condición de simetría. Por lo tanto, la ecuación del potencial de acoplamiento a ser resuelta, es expresa por:

$$
\begin{aligned}
& \frac{\partial \phi_{f o}^{*}}{\partial \tau}+\frac{\partial\left(u_{r} \phi_{f o}^{*}\right)}{\partial r}+\frac{\partial\left(u_{z} \phi_{f o}^{*}\right)}{\partial z} \\
& =\frac{1}{P e}\left[\frac{1}{r} \frac{\partial}{\partial r}\left(r \frac{\partial \phi_{f o o}^{*}}{\partial r}\right)+\frac{\partial}{\partial z}\left(\frac{\partial \phi_{f o}^{*}}{\partial z}\right)\right]
\end{aligned}
$$

Después de resuelto el potencial de acoplamiento, la solución del fenómeno reactivo es para el Oxidante $\phi_{f o}^{*} \leq \phi_{f o, s t}^{*}$ : 


$$
\begin{gathered}
Y_{f}=0 \\
Y_{o}=-s \cdot \phi_{f o} \\
\theta=-\phi_{t f}
\end{gathered}
$$

y para el Combustible $\phi_{f o}^{*}>\phi_{f o, s t}^{*}$ :

$$
\begin{gathered}
Y_{f}=\phi_{f o} \\
Y_{o}=0 \\
\theta=-\phi_{t o}
\end{gathered}
$$

El Método de los Volúmenes Finitos es aplicado para discretizar las ecuaciones de Navier-Stokes y la del Potencial de Acoplamiento. El esquema potencial WUDS es aplicado para la discretización de los términos convectivos. Entretanto, los términos difusivos son discretizados por el esquema de diferencias centradas y el esquema implícito es aplicado para la integración temporal. Todo lo relacionado con la transformación de coordenadas e integración de las ecuaciones puede ser visto en Maliska [9].

\section{RESULTADOS}

\section{Malla}

A continuación, la figura 5 presenta la malla para la geometría de la cámara de combustión propuesta. El radio de la esfera es de 0,2. El tamaño de la malla es (152x44) volúmenes. Entretanto, la figura 6 muestra un detalle en la región de la esfera donde se observa la ortogonalidad de las líneas de la malla en los contornos de la esfera y la superficie del cilindro. En general, la calidad de los elementos de la malla es bastante buena, sin embargo, se observa una región conflictiva en $x=0,8$ y $x=1,2$ para $y=0$ debido al tipo de coordenadas adoptado en el presente trabajo. Un sistema de coordenadas más adecuado para estas regiones se muestra en la figura 7 [8]. Sin embargo, la mejora de los elementos de la malla en los puntos conflictivos trae consigo una alta distorsión de los elementos de la malla a medida que éstos se alejan de la superficie de la esfera.

\section{Validación y Campos}

A continuación se presenta la validación de los resultados. Los resultados son comparados con los obtenidos por Pacheco y Leiroz [8], quienes resolvieron las ecuaciones de movimiento a través de la formulación Vorticidad-
Función Corriente con el Método de Diferencias Finitas, y con las coordenadas del tipo mostradas en la figura 7. Las secciones analizadas son mostradas en la figura 8, y fueron escogidas por ser éstas donde se presentaban los mayores conflictos. Estas secciones corresponden a la región de la esfera en contacto con las corrientes de aire primario, aire secundario y el chorro de combustible. El número de Reynolds simulado es de 750, y el número de Prandtl es fijado a 0,7 [10]. El radio de la esfera inyectora es de 0,2 . En la tabla 1 se presentan las condiciones de contorno fijadas.

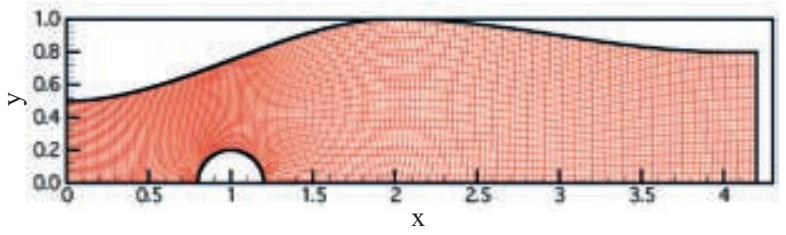

Figura 5. Malla 152x44.

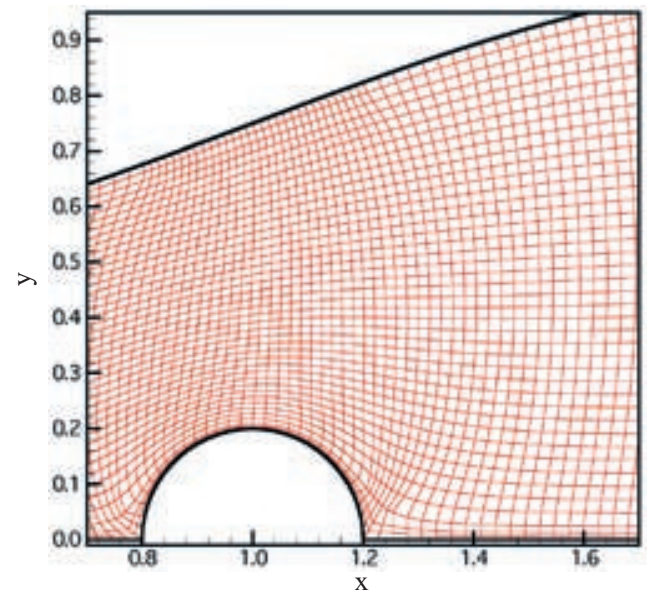

Figura 6. Detalle de la malla en la región de la esfera, coordenadas del tipo rectangular.

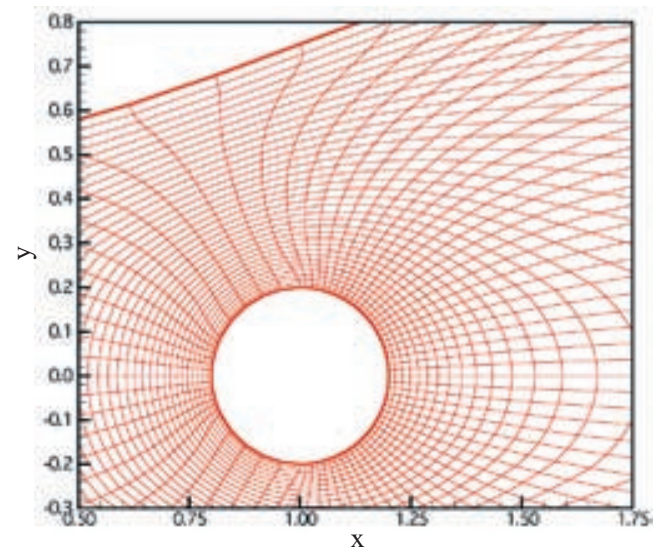

Figura 7. Detalle de la malla en la región de la esfera, coordenadas del tipo polar. 


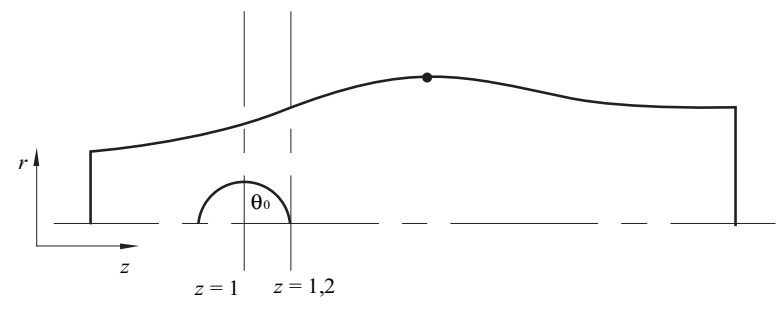

Figura 8. Secciones de análisis.

Tabla 1. Condiciones de contorno.

\begin{tabular}{|c|c|c|c|c|c|}
\hline $\boldsymbol{U}_{\boldsymbol{0}}$ & $\boldsymbol{U}_{\boldsymbol{s e c}}$ & $\boldsymbol{z}_{\boldsymbol{1}}$ & $\boldsymbol{z}_{\boldsymbol{2}}$ & $\boldsymbol{U}_{\text {jato }}$ & $\boldsymbol{\theta}_{0}$ \\
\hline 1 & 1,35 & 1,25 & 1,55 & 2 & 1 \\
\hline
\end{tabular}

La figura 9 muestra los resultados en la sección $z=1$, para la velocidad axial y radial, respectivamente. La concordancia cualitativa de los resultados es buena para ambos perfiles. Para la velocidad radial la diferencia cualitativa es notoria en la región cercana al cilindro, para $r>0,6$. Entretanto, la diferencia absoluta en esta región es de 0,02 para la velocidad radial en $r=0,65$. El criterio de diferencia relativa resulta poco aplicable, debido a que los valores de velocidad radial están próximos de cero. Entretanto, para la velocidad axial, la máxima diferencia relativa es de $16 \%$ en $r=0,28$.
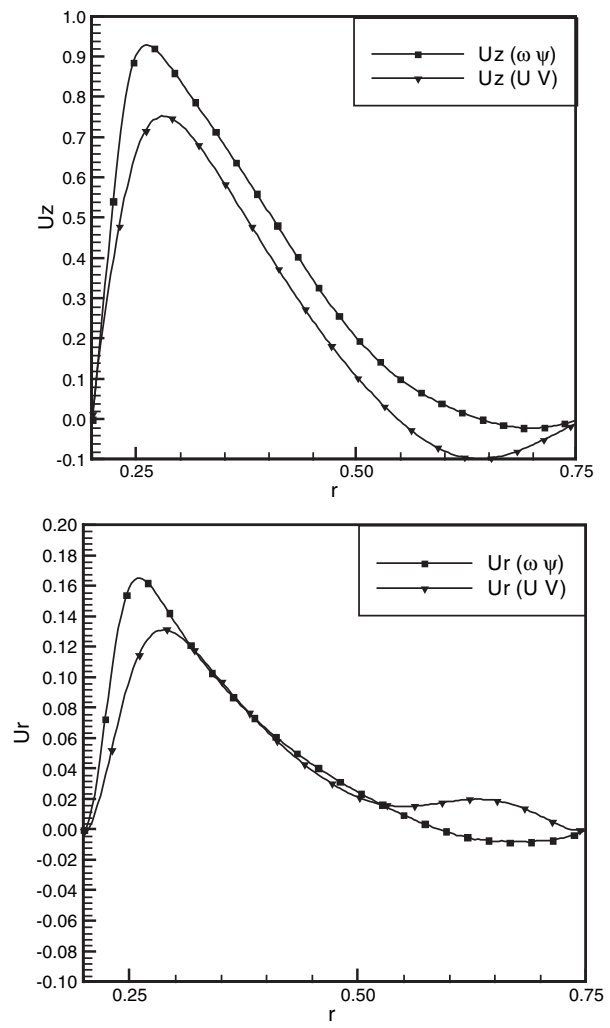

Figura 9. Velocidad Axial (arriba), Velocidad Radial (abajo), $z=1$.
La figura 10 presenta el resultado de la velocidad axial únicamente, y del potencial de acoplamiento en $z=12$. La concordancia cualitativa de los resultados para ambos perfiles es bastante buena. Las diferencias en la velocidad axial se observan en $r=0$, y es atribuido principalmente a la calidad de la malla en dicha región. Ver figura 6, y naturalmente a la naturaleza del método numérico de solución. El perfil del potencial de acoplamiento muestra una mayor difusión para los resultados obtenidos con el abordaje de variables primitivas.

Observado los campos de velocidad y del potencial de acoplamiento, concentrados en las secciones analizadas, en las figuras 11 y 12 , se observa que los resultados obtenidos con el abordaje de variables primitivas, presenta zonas de recirculación de mayor tamaño en la región anterior a la entrada del aire secundario. También se observa que la difusión del potencial de acoplamiento es mayor en la dirección radial.

La zona de recirculación es producto del fenómeno de separación de la Capa Limite, debido al aumento de área en esta región, y a la baja energía cinética que presenta el campo de velocidades en esta región.
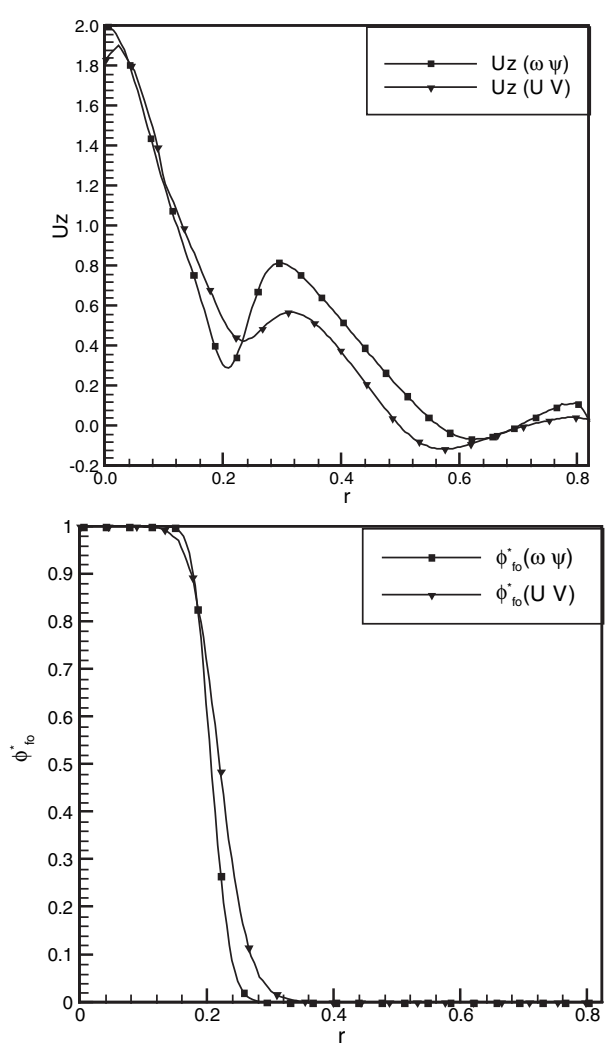

Figura 10. Velocidad Axial (arriba), Potencial de Acoplamiento (abajo), $z=1,2$. 


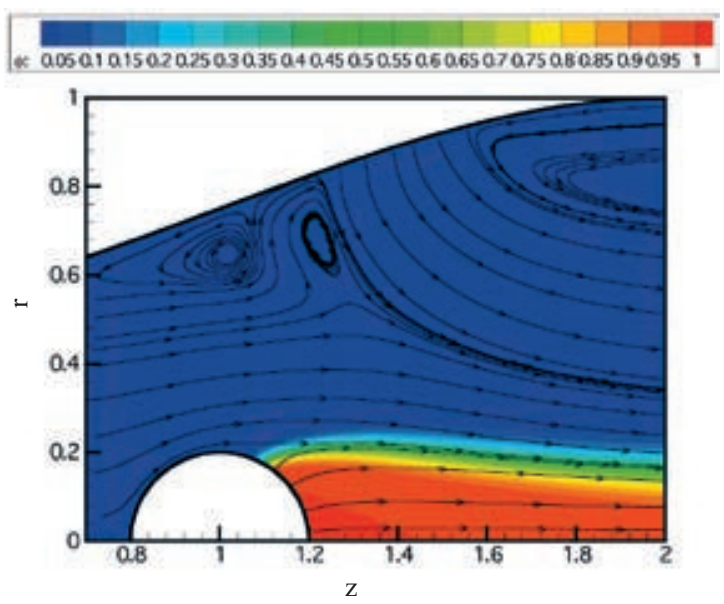

Figura 11. Potencial y Líneas de Corriente (Abordaje $\omega$ у $\psi$ ).

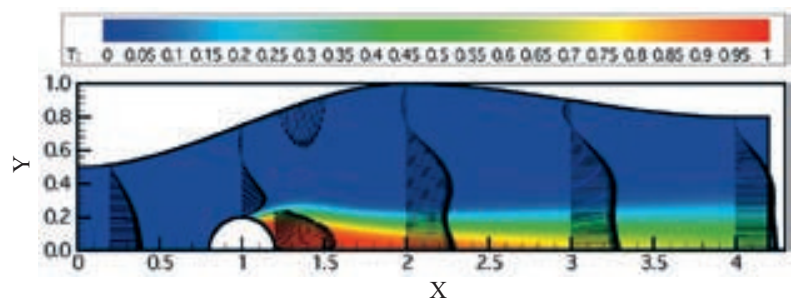

Figura 13. Potencial de Acoplamiento y Vectores de Velocidad.

La figura 13 presenta los campos del potencial de acoplamiento para todo el dominio, en conjunto con los vectores de velocidad, en secciones representativas del dominio para observar la evolución del campo de velocidades. Se observa que en la región posterior de la esfera, los máximos de velocidad se atenúan corriente abajo, debido a los efectos de roce viscoso. Por otro lado, el transporte del Potencial de Acoplamiento aumenta en la dirección radial corriente abajo, debido a que la convección disminuye, y la difusión aumenta, tanto en la dirección axial como radial. Por lo tanto, el mecanismo de transporte dominante de combustible y oxidante a la zona de reacción es el de difusión radial.

La figura 14 muestra la posición de la llama, a través de la superficie estequiométrica. El valor de esta isosuperficie se encuentra por medio de la ecuación (24). La reacción considerada es la de Metano con Aire, donde la relación aire-combustible estequiométrica es $s=17,16\left[\mathrm{Kg}\right.$ de Aire $\left./ \mathrm{Kg} \mathrm{CH}_{4}\right], Y_{O_{2}}=0,223$, por lo tanto, el valor de la iso-superficie es $\phi_{f o, s t}^{*}=0,055$. De la figura 14 se observa que la llama sale del dominio de la cámara

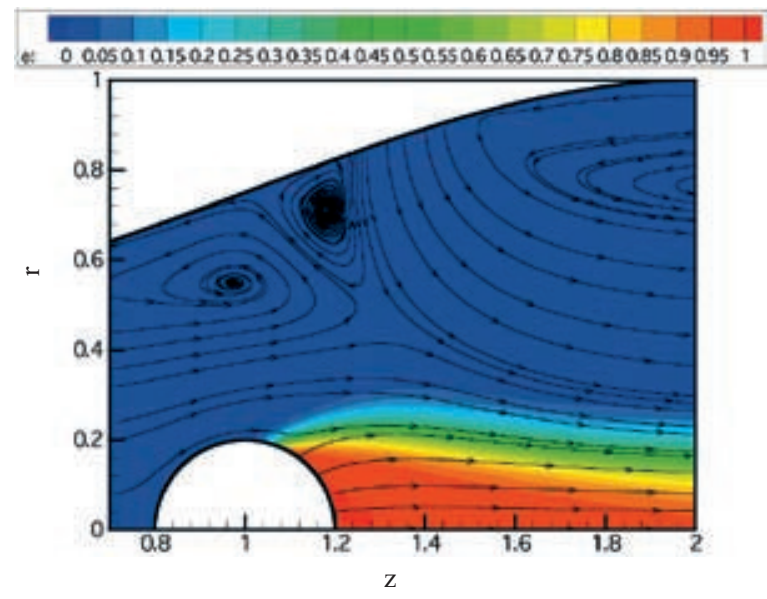

Figura 12. Potencial y Líneas de Corriente (Abordaje Variables primitivas).

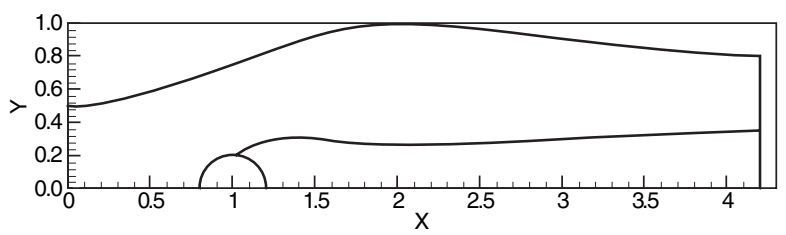

Figura 14. Posición de la Llama.

de combustión y, como consecuencia, combustible sin quemar es encontrado a la salida de la cámara. Esto se debe principalmente a que el transporte de combustible y oxidante a la zona de reacción se produce principalmente por difusión molecular. Por lo tanto, se hace necesario la presencia de efectos turbulentos para optimizar la mezcla y la quema del combustible. De esta forma, se justifica el porqué la combustión debe realizarse necesariamente en régimen turbulento, como así también, otros problemas de ingeniería en donde se procura una rápida mezcla o transporte de calor.

Resultados adicionales, para un problema con radio de esfera mayor, $r_{e s f}=0,4$, y para las mismas condiciones de contornos mostradas en la tabla 1, se presentan de las figuras 15 a 17. De la figura 15 se observa que la mayor diferencia en relación al caso anterior reside en la ausencia de la gran zona de recirculación en $z=1$. La justificación consiste en que el área de paso de fluido es menor, como consecuencia del mayor radio de la esfera $\mathrm{y}$, por lo tanto, la energía cinética del fluido es mayor y las probabilidades de que la capa límite sufra separación resultan menores. 


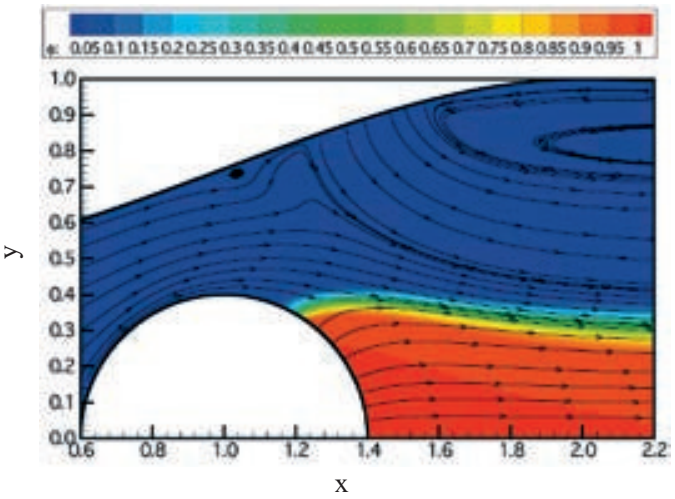

Figura 15. Potencial y líneas de corriente, $r_{e s f}=0,4$.

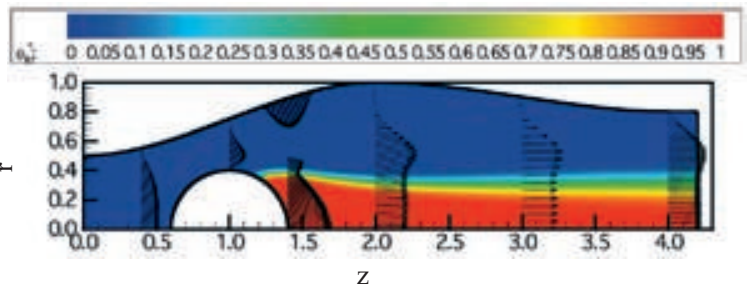

Figura 16. Potencial de acoplamiento y vectores de Velocidad, $r_{\text {esf }}=0,4$.

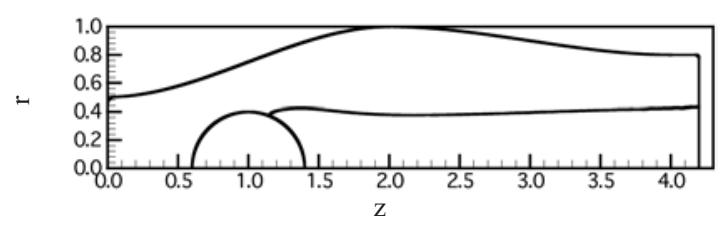

Figura 17. Posición de la llama, $r_{e s f}=0,4$.

\section{CONCLUSIONES}

Se ha presentado un estudio que permite la simulación de flujos reactivos al interior de una cámara de combustión, en variables primitivas de acuerdo con el Método de Volúmenes Finitos, algoritmo SIMPLEC. Entretanto, el fenómeno reactivo fue tratado a través de la formulación Shvab-Zel'dovich, bajo la hipótesis de llama fina y un número de Lewis unitario para combustible y oxidante.

Los resultados fueron validados con resultados obtenidos numéricamente basados en la formulación de VorticidadFunción Corriente. Existen diferencias menores entre los resultados obtenidos por formulación Vorticidad-Función Corriente en relación a formulación en variables primitivas abordados según el algoritmo SIMPLEC.
La formulación de Shvab-Zel'dovich se ha mostrado efectiva para la determinación de la forma de la llama, pero no para la distribución de temperaturas.

\section{REFERENCIAS}

[1] Kuo Kenneth. "Principles of Combustion". John Wiley \& Sons Inc. Primera edición. 1986.

[2] V.R. Katta, L.P. Goss y W.M. Roquemore. "Effect og Nonunity Lewis Number and Finite-Rate Chemistry on the Dynamics of a Hydrogen-Air Jet Diffusion Flame". Combustion and Flame. Vol. 96, pp. 60-74. 1994.

[3] S. Menom, V. Sankaran y C. Stone. "Dynamics of Swirling Premixed and Spray Flames". AIAA 2001-1092. 2001.

[4] F. di Mare, W.P. Jones y K.R. Menzies. "Large Eddy simulation of a model gas turbine combustor". Combustion and Flame. Vol.137, pp. 278-294. 2004.

[5] L. Selle, G. Lartigue, T. Poinsot, R. Koch, K. Schildmacher, W. Krebs, B. Prade, P. Kaufmann y D. Veynante. "Compressible large eddy simulation of turbulent combustion in complex geometry on unstructured meshes", Combustion and Flame. Vol. 137, pp. 489-505. 2004.

[6] M. Özisik, Necati. "Finite Difference Methods in Heat Transfer”. Editora CRC Press, Boca Raton. Primera Edición 1994.

[7] J.D. Hofmann. "Numerical Method for Engineers and Scientists". McGraw-Hill inc. 1992.

[8] P.A. Pacheco. "Escoamentos Reativos em Geometrias-Modelo de Câmaras de Combustão de Turbinas a Gás". Tesis para optar al grado e Magíster. Instituto Alberto Luiz Coimbra. Coordenação de Aperfeiçoamento de Pessoal de Nível Superior. Rio de Janeiro, Brasil. 2005.

[9] C.R. Maliska. "Transferência de Calor e Mecânica dos Fluidos Computacional”. $2^{\circ}$ Edição. Revista e Ampliada. Rio de Janeiro, Brasil. Editora LTC. 2004.

[10] J.H. Lienhard IV, J.H.Lienhard V. "A Heat Transfer TextBook". Cambridge MA: Phlogiston Press. Third edition. 2003. 